\title{
The open mapping theorem for regular quaternionic functions
}

\author{
GraZiano Gentili ANd CATERINA Stoppato
}

\begin{abstract}
The basic results of a new theory of regular functions of a quaternionic variable have been recently stated, following an idea of Cullen. In this paper we prove the minimum modulus principle and the open mapping theorem for regular functions. The proofs involve some peculiar geometric properties of such functions which are of independent interest.
\end{abstract}

Mathematics Subject Classification (2000): 30G35 (primary).

\section{Introduction}

Let $\mathbb{H}$ denote the skew field of real quaternions. Its elements are of the form $q=$ $x_{0}+i x_{1}+j x_{2}+k x_{3}$ where the $x_{l}$ are real, and $i, j, k$, are imaginary units (i.e. their square equals -1 ) such that $i j=-j i=k, j k=-k j=i$, and $k i=-i k=j$.

After Hamilton's discovery of quaternions, the richness of the theory of holomorphic functions of one complex variable, along with motivations from physics, aroused interest in a theory of quaternion-valued functions of a quaternionic variable. In fact several interesting theories have been introduced in the last century. The most famous is the theory of regular functions introduced by R. Fueter, [4,5], the basic results of which are accurately summarized in [16]. Recent work on this subject includes $[1,10]$ and references therein. Fueter also introduced the class of holomorphic functions over quaternions, to which the works of M. Sce $[3,15]$ are closely related. The latest advances in this theory (which extends to all Clifford algebras) can be found in $[9,11,12,14]$.

A different theory of quaternion-valued functions of one quaternionic variable has been recently proposed by G. Gentili and D. C. Struppa [7, 8]. The theory is based on a definition of regularity for quaternionic functions inspired by $\mathrm{C}$. G. Cullen [2]. Several basic results of the theory are proven in [8], including the

Both authors were partially supported by GNSAGA of the INdAM and by PRIN "Proprietà geometriche delle varietà reali e complesse" of the MIUR. The second was also partially supported by PRIN "Geometria Differenziale e Analisi Globale" of the MIUR.

Received September 24, 2008; accepted in revised form March 9, 2009. 
Cullen-regularity of quaternion power series and some nice properties of their zeros. The study of the zero-sets has been further developed in [6].

We shall now quickly review the definition of Cullen-regular function and the basic properties of such a function. Denote by $\mathbb{S}$ the two-dimensional sphere of quaternion imaginary units: $\mathbb{S}=\left\{q \in \mathbb{H}: q^{2}=-1\right\}$. For all imaginary unit $I \in \mathbb{S}$, let $L_{I}=\mathbb{R}+I \mathbb{R}$ be the complex line through 0,1 and $I$.

Definition 1.1. Let $\Omega$ be a domain in $\mathbb{H}$ and let $f: \Omega \rightarrow \mathbb{H}$ be a real differentiable function. $f$ is said to be Cullen-regular if, for all $I \in \mathbb{S}$, the function $\bar{\partial}_{I} f: \Omega \cap$ $L_{I} \rightarrow \mathbb{H}$ defined by

$$
\bar{\partial}_{I} f(x+I y)=\frac{1}{2}\left(\frac{\partial}{\partial x}+I \frac{\partial}{\partial y}\right) f_{I}(x+I y)
$$

vanishes identically.

With the notations $\Omega_{I}=\Omega \cap L_{I}$ and $f_{I}=f_{\Omega_{\Omega_{I}}}$, we may refer to the vanishing of $\bar{\partial}_{I} f$ saying that the restriction $f_{I}$ is holomorphic on $\Omega_{I}$. From now on we will omit Cullen's name and refer to these functions just as regular functions. As observed in [8], a quaternionic power series $\sum_{n \in \mathbb{N}} q^{n} a_{n}$ with $a_{n} \in \mathbb{H}$ defines a regular function in its domain of convergence, which proves to be an open ball $B(0, R)=\{q \in \mathbb{H}:|q|<R\}$. In the same paper, it is proven that:

Theorem 1.2. If $f: B=B(0, R) \rightarrow \mathbb{H}$ is regular then there exist quaternions $a_{n} \in \mathbb{H}$ such that

$$
f(q)=\sum_{n \in \mathbb{N}} q^{n} a_{n}
$$

for all $q \in B$. In particular, $f \in C^{\infty}(B)$.

Many classical results in complex analysis are extended in [8] to regular functions $f: B(0, R) \rightarrow \mathbb{H}$ : the identity principle, the maximum modulus principle, the Cauchy representation formula, the Liouville theorem, the Morera theorem and the Schwarz lemma.

In this paper we prove the open mapping theorem for regular functions. We point out that this result is straightforward in the special case of series $f(q)=$ $\sum_{n \in \mathbb{N}} q^{n} a_{n}$ having real coefficients $a_{n} \in \mathbb{R}$ (series which are also studied in [3,9, 12]): it is easy to deduce that such an $f$ is open from the open mapping theorem for holomorphic functions of one complex variable. The general proof is elaborate, and it requires several preliminary steps.

We first define an operation on regular functions which allows us to deduce a weak version of the minimum modulus principle (see Theorem 4.3) from the maximum modulus principle proven in [8]. We then study the behavior of a regular function $f$ restricted to any 2 -sphere $S=x+y \mathbb{S}(x, y \in \mathbb{R}, y \neq 0)$ and find out that $f_{\mid S}$ is either constant or an affine map of $S$ onto a 2 -sphere $b+\mathbb{S} c$ with $b, c \in \mathbb{H}$. This peculiar geometric property, which is of independent interest, leads to the following version of the minimum modulus principle: 
Theorem 1.3 (Minimum modulus principle). Let $f: B=B(0, R) \rightarrow \mathbb{H}$ be $a$ regular function. If $|f|$ has a local minimum point $p \in B$ then either $f(p)=0$ or $f$ is constant.

We define the degenerate set $D_{f}$ of $f$ as the union of all the 2-spheres $S=$ $x+y \mathbb{S}(x, y \in \mathbb{R}, y \neq 0)$ on which $f$ is constant. If $f$ is not constant then $D_{f}$ turns out to have empty interior and by means of the above result we prove:

Theorem 1.4 (Open mapping theorem). Let $f: B(0, R) \rightarrow \mathbb{H}$ be a non-constant regular function and let $D_{f}$ be its degenerate set. Then $f: B(0, R) \backslash \overline{D_{f}} \rightarrow \mathbb{H}$ is open.

Corollary 1.5. Let $f: B(0, R) \rightarrow \mathbb{H}$ be a regular function. If $f$ is not constant on any 2 -sphere $x+y \mathbb{S}$ with $y \neq 0$, then $f$ is open.

We give an example of regular function $f: B=B(0, R) \rightarrow \mathbb{H}$ which is open on $B \backslash \overline{D_{f}}$ but not on $B$. Finally, we show that an open subset $U \subseteq B(0, R)$ such that $U=\bigcup_{x+y I \in U} x+y \mathbb{S}$ always maps to an open set $f(U)$, even when $U$ intersects the degenerate set $D_{f}$. In particular $f(B(0, R))$ is always an open set.

\section{Preliminary results}

We will recall in this section some results and definitions which appear in the study of the structure of the zero-sets of regular functions presented in $[6,8]$. We begin with a symmetry property of the zeros proven in [8]. This result and the following are proven for polynomials in [13].

Theorem 2.1. Let $f: B=B(0, R) \rightarrow \mathbb{H}$ be a regular function and let $x, y \in$ $\mathbb{R}, y>0$ be such that $x^{2}+y^{2}<R^{2}$. If there exist distinct imaginary units $I, J \in \mathbb{S}$ such that $f(x+y I)=f(x+y J)=0$, then $f(x+y K)=0$ for all $K \in \mathbb{S}$.

In other words, if $f$ has more than one zero on the 2 -sphere $x+y \mathbb{S}$ then it vanishes identically on $x+y \mathbb{S}$. In [6] the zero-set is further characterized as follows.

Theorem 2.2. Let $f$ be a regular function on an open ball $B=B(0, R)$. If $f$ is not identically zero then its zero-set consists of isolated points or isolated 2-spheres of the form $x+y \mathbb{S}$, for $x, y \in \mathbb{R}, y \neq 0$.

The study of the zeros presented in [6] involves the following operations on regular functions $f: B(0, R) \rightarrow \mathbb{H}$.

Definition 2.3. Let $f, g$ be regular functions on an open ball $B=B(0, R)$ and let $f(q)=\sum_{n \in \mathbb{N}} q^{n} a_{n}, g(q)=\sum_{n \in \mathbb{N}} q^{n} b_{n}$ be their power series expansions. We define the regular product of $f$ and $g$ as the regular function $f * g: B \rightarrow \mathbb{H}$ with

$$
f * g(q)=\sum_{n \in \mathbb{N}} q^{n} c_{n}, \quad c_{n}=\sum_{k=0}^{n} a_{k} b_{n-k} .
$$


We define the regular conjugate of $f$ as the function $f^{c}: B \rightarrow \mathbb{H}$, satisfying the following formula: $f^{c}(q)=\sum_{n \in \mathbb{N}} q^{n} \bar{a}_{n}$. Finally, we define the symmetrization of $f$ as $f^{s}=f * f^{c}=f^{c} * f$.

Note that the regular multiplication is an associative, non-commutative binary operation. Since no confusion can arise, we may also write $f(q) * g(q)$ for $f *$ $g(q)$. Also note that the symmetrization $f^{s}(q)=\sum_{n \in \mathbb{N}} q^{n} \sum_{k=0}^{n} a_{k} \bar{a}_{n-k}$ has real coefficients. The zero-sets of $f^{c}$ and $f^{s}$ are characterized in [6] as follows.

Theorem 2.4. Let $f$ be a regular function on $B=B(0, R)$. For all $x, y \in \mathbb{R}$ with $x+y \mathbb{S} \subseteq B$, the zeros of the regular conjugate $f^{c}$ on $x+y \mathbb{S}$ are in one-to-one correspondence with those of $f$. Moreover, the symmetrization $f^{s}$ vanishes exactly on the sets $x+y \mathbb{S}$ on which $f$ has a zero.

Note that $x+y \mathbb{S}$ is a 2 -sphere if $y \neq 0$ and a real singleton $\{x\}$ if $y=0$.

\section{The reciprocal function}

We define a new operation on regular functions, associating to any regular $f$ : $B(0, R) \rightarrow \mathbb{H}$ a function $f^{-*}$ called its regular reciprocal. The reason for this name will become apparent later. Denote by $\mathcal{Z}_{f}$ the zero set of a regular function $f$.

Definition 3.1. Let $f: B=B(0, R) \rightarrow \mathbb{H}$ be a regular function and let $f^{c}, f^{s}$ be its regular conjugate and symmetrization. We call the regular reciprocal of $f$ the function $f^{-*}: B \backslash \mathcal{Z}_{f^{s}} \rightarrow \mathbb{H}$ defined by

$$
f^{-*}(q)=\frac{1}{f^{s}(q)} f^{c}(q) .
$$

We will use the shorthand notation $f^{-s}(q)$ for $\frac{1}{f^{s}(q)}$. Also, from now on we will refer to $f^{-*}$ just as the reciprocal of $f$, omitting the adjective "regular" for the sake of simplicity. The reciprocal $f^{-*}$ of a regular function is regular due to the following result, which can be proven by direct computation.

Lemma 3.2. Let $f, g: B=B(0, R) \rightarrow \mathbb{H}$ be regular functions and suppose the power series expansion of $g$ at $0, g(q)=\sum_{n \in \mathbb{N}} q^{n} r_{n}$, has real coefficients $r_{n} \in \mathbb{R}$. Then the function $h: B \backslash \mathcal{Z}_{g} \rightarrow \mathbb{H}$ defined by $h(q)=\frac{1}{g(q)} f(q)$ is regular.

If $f$ does not have any zeros then $f^{-*}$ is the inverse element of the function $f$ with respect to regular multiplication:

Proposition 3.3. Let $f$ be a regular function on $B=B(0, R)$ which does not have zeros. Then $f^{-*}$ is a regular function on $B$ and

$$
f * f^{-*}=f^{-*} * f=1 .
$$


Proof. By Theorem 2.4, if $f$ does not have zeros then the zero set $\mathcal{Z}_{f}$ of its symmetrization is empty. In this case $f^{-s}$ and $f^{-*}$ are well defined and regular on the whole ball $B$ and we may consider their regular product with other regular functions $g: B \rightarrow \mathbb{H}$. We first note that for all such $g$ we have $f^{-s}(q) g(q)=$ $f^{-s}(q) * g(q)=g(q) * f^{-s}(q)$. We may then compute:

$$
\begin{aligned}
& f^{-*} * f=f^{-s} * f^{c} * f=f^{-s} f^{s}=1, \\
& f * f^{-*}=f * f^{-s} * f^{c}=f^{-s} * f * f^{c}=f^{-s} f^{s}=1 .
\end{aligned}
$$

We now give an alternative expression of the regular product and the reciprocal.

Proposition 3.4. Let $f, g$ be regular functions on $B=B(0, R)$. Then

$$
f * g(q)=f(q) g\left(f(q)^{-1} q f(q)\right)
$$

for all $q \in B \backslash \mathcal{Z}_{f}$.

Proof. Let $f(q)=\sum_{n \in \mathbb{N}} q^{n} a_{n}, g(q)=\sum_{n \in \mathbb{N}} q^{n} b_{n}$ be the power series expansions of $f$ and $g$. By Definition 2.3,

$$
f * g(q)=\sum_{n \in \mathbb{N}} q^{n} \sum_{k=0}^{n} a_{k} b_{n-k}=\sum_{k, l \in \mathbb{N}} q^{l+k} a_{k} b_{l}=\sum_{l \in \mathbb{N}} q^{l} f(q) b_{l} .
$$

If $f(q) \neq 0$ then

$$
\begin{aligned}
\sum_{l \in \mathbb{N}} q^{l} f(q) b_{l} & =\sum_{l \in \mathbb{N}} f(q) f(q)^{-1} q^{l} f(q) b_{l}=f(q) \sum_{l \in \mathbb{N}}\left[f(q)^{-1} q f(q)\right]^{l} b_{l} \\
& =f(q) g\left(f(q)^{-1} q f(q)\right) .
\end{aligned}
$$

Proposition 3.5. Let $f$ be a regular function on $B=B(0, R)$. If we set $T_{f}(q)=$ $f^{c}(q)^{-1} q f^{c}(q)$, then

$$
f^{-*}(q)=f\left(T_{f}(q)\right)^{-1}
$$

for all $q \in B \backslash \mathcal{Z}_{f^{s}}$.

Proof. If $f^{s}(q) \neq 0$ then $f^{c}(q) \neq 0$, hence $T_{f}$ is well defined on $B \backslash \mathcal{Z}_{f}$. We also note that, by the previous result, $f^{c}(q) * g(q)=f^{c}(q) g\left(T_{f}(q)\right)$. We conclude by computation:

$$
\begin{aligned}
f^{-*}(q) & =f^{s}(q)^{-1} f^{c}(q)=\left[f^{c} * f(q)\right]^{-1} f^{c}(q) \\
& =\left[f^{c}(q) f\left(T_{f}(q)\right)\right]^{-1} f^{c}(q)=f\left(T_{f}(q)\right)^{-1} f^{c}(q)^{-1} f^{c}(q) \\
& =f\left(T_{f}(q)\right)^{-1}
\end{aligned}
$$


This result clarifies, together with proposition 3.3, why we call $f^{-*}$ the "reciprocal" of $f$. Note that $T_{f}: B \backslash \mathcal{Z}_{f^{s}} \rightarrow \mathbb{H}$ maps any 2 -sphere (or real singleton) $x+y \mathbb{S}$ to itself. In particular $T_{f}\left(B \backslash \mathcal{Z}_{f^{s}}\right) \subseteq B \backslash \mathcal{Z}_{f^{s}}$. Moreover, we prove the following:

Proposition 3.6. Let $f: B=B(0, R) \rightarrow \mathbb{H}$ be a regular function. $T_{f}$ and $T_{f c}$ are mutual inverses. In particular $T_{f}: B \backslash \mathcal{Z}_{f} \rightarrow B \backslash \mathcal{Z}_{f^{s}}$ is a diffeomorphism.

Proof. For all $q \in B \backslash \mathcal{Z}_{f}$ we have, setting $p=T_{f}(q)$,

$$
\begin{aligned}
T_{f^{c}} \circ T_{f}(q) & =T_{f^{c}}(p)=f(p)^{-1} p f(p) \\
& =f(p)^{-1}\left[f^{c}(q)^{-1} q f^{c}(q)\right] f(p)=\left[f^{c}(q) f(p)\right]^{-1} q\left[f^{c}(q) f(p)\right]
\end{aligned}
$$

where

$$
f^{c}(q) f(p)=f^{c}(q) f\left(f^{c}(q)^{-1} q f^{c}(q)\right)=f^{c} * f(q)=f^{s}(q) .
$$

Hence

$$
T_{f^{c}} \circ T_{f}(q)=f^{s}(q)^{-1} q f^{s}(q)=q,
$$

where the last equality holds because $f^{s}(q)$ always lies in the same complex line as $q$ so that they commute.

\section{The minimum modulus principle}

The reciprocal function will allow us to derive the minimum modulus principle from the maximum modulus principle proven in [8]. Actually, the latter only applies to functions which are regular on a ball $B(0, R)$. In order to apply it to the reciprocal $f^{-*}: B \backslash \mathcal{Z}_{f^{s}} \rightarrow \mathbb{H}$ we need to extend the maximum modulus principle to a slightly larger class of functions. We first extend the identity principle proven in [8].

Proposition 4.1 (Identity principle). Let $\Omega$ be a domain in $\mathbb{H}$ intersecting the real axis and having connected intersection $\Omega_{I}=\Omega \cap L_{I}$ with any complex line $L_{I}$. If $f, g: \Omega \rightarrow \mathbb{H}$ are regular functions which coincide on $\Omega \cap \mathbb{R}$ then they coincide on the whole domain $\Omega$.

Proof. Let $h=f-g$ and let us prove $h \equiv 0$ on $\Omega$. Choose any imaginary unit $I \in \mathbb{S}$ and consider the restriction $h_{I}=h_{\left.\right|_{\Omega_{I}}}$. Since $h_{I}: \Omega_{I} \rightarrow \mathbb{H}$ is holomorphic and it vanishes on the set $\Omega \cap \mathbb{R}$, which is not discrete, $h_{I}$ must vanish identically on $\Omega_{I}$.

We are now ready to extend the maximum modulus principle as desired.

Theorem 4.2 (Maximum modulus principle). Let $\Omega$ be the quaternionic ball $B=$ $B(0, R)$ minus a (closed) set $\mathcal{E}$ consisting of isolated points and isolated 2-spheres $x+y \mathbb{S}$. Let $f: \Omega \rightarrow \mathbb{H}$ be a regular function. If $|f|$ has a local maximum point $p \in \Omega$ then $f$ is constant in $\Omega$. 
Proof. Let $L_{I}$, with $I \in \mathbb{S}$, be the complex line through $p$ in $\mathbb{H}$ and consider the restriction $f_{I}: \Omega_{I} \rightarrow \mathbb{H}$. If $p$ is a maximum point for $|f|$ then it is also a maximum point for $\left|f_{I}\right|$. By the same argument used in [8], we conclude $f_{I}$ is constant on $\Omega_{I}$. By the identity principle $4.1, f$ is constant on $\Omega$.

Theorem 4.3 (Weak minimum modulus principle). Let $f: B=B(0, R) \rightarrow \mathbb{H}$ be a regular function. If $|f|$ has a local minimum point $p=x+y I \in B$ then either $f$ is constant or $f$ has a zero in $x+y \mathbb{S}$.

Proof. Suppose $f$ does not have zeroes in $S=x+y \mathbb{S}$ and consider the reciprocal $f^{-*}$ : it is defined on the domain $\Omega=B \backslash \mathcal{Z}_{f^{s}}$, which includes $S$. Note that

$$
\left|f^{-*}(q)\right|=\frac{1}{\left|f\left(T_{f}(q)\right)\right|}
$$

for all $q \in \Omega$. Since $T_{f}$ is a diffeomorphism of $B \backslash \mathcal{Z}_{f^{s}}$ onto itself, the fact that $|f|$ has a minimum at $p=T_{f}\left(p^{\prime}\right)$ implies that $\left|f \circ T_{f}\right|$ has a minimum at $p^{\prime}$. As a consequence, $\left|f^{-*}\right|$ has a maximum at $p^{\prime}$. By the maximum modulus principle 4.2, $f^{-*}$ is constant on $\Omega$. This implies that $f$ is constant, too.

We will soon prove a stronger version of the minimum modulus principle, which will allow us to prove the open mapping theorem for regular quaternionic functions.

\section{Value distribution on the 2-spheres}

We now want to strengthen the minimum modulus principle, proving that if the modulus of a non-constant regular function $f$ has a minimum at a non-real point $p=x+y I$ then $p$ itself must be a zero for $f$. In order to prove this result, we need to better understand the distribution of the values of $f$ on the 2 -sphere $x+y \mathbb{S}$ through $p$.

Theorem 5.1. Let $f: B=B(0, R) \rightarrow \mathbb{H}$ be a regular function. For all $x, y \in \mathbb{R}$ such that $x+y \mathbb{S} \subseteq B$ there exist $b, c \in \mathbb{H}$ such that

$$
f(x+y I)=b+I c
$$

for all $I \in \mathbb{S}$. Note that $c=0$ if, and only if, $f$ is constant on $x+y \mathbb{S}$.

Proof. Let $f(q)=\sum_{n \in \mathbb{N}} q^{n} a_{n}$ be the power series expansion of $f$ at 0 and, for all $n \in \mathbb{N}$, let $x_{n}, y_{n} \in \mathbb{R}$ be such that $(x+y I)^{n}=x_{n}+y_{n} I$. Then

$$
\begin{aligned}
f(x+y I) & =\sum_{n \in \mathbb{N}}(x+y I)^{n} a_{n}=\sum_{n \in \mathbb{N}}\left(x_{n}+y_{n} I\right) a_{n} \\
& =\sum_{n \in \mathbb{N}} x_{n} a_{n}+I \sum_{n \in \mathbb{N}} y_{n} a_{n}=b+I c .
\end{aligned}
$$


We label the 2-spheres characterized by $c=0$ with the following definition:

Definition 5.2. Let $f: B=B(0, R) \rightarrow \mathbb{H}$ be a regular function and let $x, y \in$ $\mathbb{R}, y>0$ be such that $S=x+y \mathbb{S} \subseteq B$. The 2-sphere $S$ is said to be degenerate for $f$ if the restriction $f_{\mid S}$ is constant. The union $D_{f}$ of all degenerate spheres for $f$ is called the degenerate set of $f$.

\section{Example 5.3.}

i) Let $P(q)$ be a quaternionic polynomial having real coefficients and a non-real root $x+y I$. Then $x+y \mathbb{S}$ is a degenerate sphere for $P$. A simple example is given by the polynomial $q^{2}+1$, which vanishes on the 2 -sphere $\mathbb{S}$.

ii) The regular function $f: B(0,1) \rightarrow \mathbb{H}$ defined by $f(q)=\sum_{n \in \mathbb{N}} q^{n}=\frac{1}{1-q}$ has no degenerate spheres. In fact in this case $f_{I}: B_{I} \rightarrow L_{I}$ can be written as $f_{I}(x+y I)=\frac{1-x}{(1-x)^{2}+y^{2}}+\frac{y}{(1-x)^{2}+y^{2}} I$ and $\frac{y}{(1-x)^{2}+y^{2}}=0$ if, and only if, $y=0$. Therefore there are no degenerate spheres for $f$.

If $f: B \rightarrow \mathbb{H}$ is regular and $S=x+y \mathbb{S} \subseteq B$ (with $x, y \in \mathbb{R}, y>0$ ) is not degenerate, then the restriction $f_{\left.\right|_{S}}$ is an affine map of $S$ onto a 2-sphere $b+\mathbb{S} c$ with $b, c \in \mathbb{H}$. As an immediate consequence, we get the following corollary.

Corollary 5.4. Let $f: B=B(0, R) \rightarrow \mathbb{H}$ be a regular function, let $x, y \in \mathbb{R}$ be such that $S=x+y \mathbb{S} \subseteq B$ and suppose $S$ not to be degenerate. Then $\left|f_{\mid S}\right|$ has a global minimum, a global maximum and no other extremal point.

As a consequence, we get a stronger version of the minimum modulus principle:

Theorem 5.5 (Minimum modulus principle). Let $f: B=B(0, R) \rightarrow \mathbb{H}$ be $a$ regular function. If $|f|$ has a local minimum point $p \in B$ then either $f(p)=0$ or $f$ is constant.

Proof. By Theorem 4.3 a non-constant $f$ whose modulus has a minimum at $p=$ $x+y I$ must have a zero at a point $p^{\prime} \in x+y \mathbb{S}$. In particular $|f|$ has a minimum at $p^{\prime}$. By the previous corollary, $|f|$ cannot have two distinct local minimum points on $x+y \mathbb{S}$, unless $x+y \mathbb{S}$ is degenerate for $f$. As a consequence, either $f$ is constant on $x+y \mathbb{S}$ or $p=p^{\prime}$. In both cases, $f(p)=f\left(p^{\prime}\right)=0$.

This result will prove very useful in the next section.

\section{The open mapping theorem}

We are now ready to prove the open mapping theorem for quaternionic regular functions. Before doing so, we want to state two properties of the degenerate set of a regular function, defined in 5.2. 
Proposition 6.1. If $f: B=B(0, R) \rightarrow \mathbb{H}$ is a regular function, then the degenerate set $D_{f}$ of $f$ is a closed subset of $B \backslash \mathbb{R}$. Moreover, if $f$ is not a constant function then the interior of $D_{f}$ is empty.

Proof. By Theorem 5.1, there exist real analytic functions $b=b(x, y), c=c(x, y)$ such that $f(x+y I)=b(x, y)+I c(x, y)$. Denote by $C_{f}$ the union of all spheres (or real singletons) $x+y \mathbb{S}, x, y \in \mathbb{R}$ such that $c(x, y)=0$. We have $D_{f}=C_{f} \backslash \mathbb{R}$ since, for all $x, y \in \mathbb{R}, y \neq 0, f$ is constant on $x+y \mathbb{S}$ if and only if $c(x, y)=0$. Clearly $C_{f}$ is closed in $B$, thus $D_{f}=C_{f} \backslash \mathbb{R}$ is closed in $B \backslash \mathbb{R}$. Moreover, if $f$ is not constant then the interior of $D_{f}$ is empty because the interior of $C_{f}$ is. Assume by contradiction that, for some $I \in \mathbb{S}$, there exist a non-empty open subset $A_{I}$ of $B_{I}=B \cap L_{I}$ such that $A_{I} \subseteq C_{f}$, i.e. such that $c(x, y)=\sum_{n \in \mathbb{N}} y_{n} a_{n}=0$ for all $x+y I \in A_{I}$. If we set the obvious notation $a_{n}=a_{n}^{0}+a_{n}^{1} i+a_{n}^{2} j+a_{n}^{3} k$ then, for $p=0,1,2,3$, the holomorphic function $f_{p}: B_{I} \rightarrow L_{I}$ defined by

$$
f_{p}(x+y I)=\sum_{n \in \mathbb{N}}(x+y I)^{n} a_{n}^{p}=\sum_{n \in \mathbb{N}} x_{n} a_{n}^{p}+\sum_{n \in \mathbb{N}} y_{n} a_{n}^{p} I
$$

has vanishing imaginary part on the open set $A_{I}$. Thus $f\left(A_{I}\right) \subseteq \mathbb{R}$ and, by the open mapping theorem for holomorphic functions of one complex variable, $f_{p}$ is constant on $B_{I}$ for $p=0,1,2,3$. Hence $f$ is constant on $B_{I}$ and, by the identity principle 4.1 , on $B$.

Theorem 6.2 (Open mapping theorem). Let $f: B(0, R) \rightarrow \mathbb{H}$ be a non-constant regular function and let $D_{f}$ be its degenerate set. Then $f: B(0, R) \backslash \overline{D_{f}} \rightarrow \mathbb{H}$ is open.

Proof. Let $U$ be an open subset of $B(0, R) \backslash \overline{D_{f}}$ and let $p_{0} \in f(U)$. We will show that the image $f(U)$ contains a ball centered at $p_{0}$ of radius $\varepsilon>0$.

Choose $q_{0} \in U$ such that $f\left(q_{0}\right)=p_{0}$. By Theorem 2.2, either the point $q_{0}$ is an isolated zero of the function $f(q)-p_{0}$, or it is part of a 2-sphere $S$ of zeros of the same function. The second possibility is excluded because it would imply $f_{\left.\right|_{S}} \equiv p_{0}$, so that $S$ would be a degenerate sphere for $f$ and $q_{0}$ would belong to the degenerate set $D_{f}$ (which is impossible by construction).

Hence $q_{0}$ is an isolated zero of $f(q)-p_{0}$ and there exists an open ball $B=$ $B\left(q_{0}, r\right)$ such that $\bar{B} \subseteq U$ and $f(q)-p_{0} \neq 0$ for all $q \in \partial B$. In particular, there exists $\varepsilon>0$ such that $\left|f(q)-p_{0}\right| \geq 3 \varepsilon$ for all $q \in \partial B$. Choosing any $p$ such that $\left|p-p_{0}\right| \leq \varepsilon$, we get

$$
|f(q)-p| \geq\left|f(q)-p_{0}\right|-\left|p-p_{0}\right| \geq 3 \varepsilon-\varepsilon=2 \varepsilon
$$

for all $q \in \partial B$, while

$$
\left|f\left(q_{0}\right)-p\right|=\left|p_{0}-p\right| \leq \varepsilon .
$$

Hence the minimum of $|f(q)-p|$ in $\bar{B}$ is strictly less than its minimum in $\partial B$, and $|f(q)-p|$ must have a minimum point in $B$. By Theorem 5.5, either $f(q)-p$ 
vanishes at the same point or it is a constant function. The second option is excluded since $f$ is not constant. We have thus proven the existence of a $q \in B \subseteq U$ such that $f(q)=p$ and we conclude that $p \in f(U)$, as desired.

If $f$ has no degenerate spheres, as in Example 5.3 ii), then we get the statement:

Corollary 6.3. Let $f: B(0, R) \rightarrow \mathbb{H}$ be a regular function. If $f$ is not constant on any 2 -sphere $x+y \mathbb{S}$ with $y \neq 0$, then $f$ is open.

We point out that there exist regular functions $f: B(0, R) \rightarrow \mathbb{H}$ which are open on $B(0, R) \backslash \overline{D_{f}}$ but not on $B(0, R)$. For instance the function $f(q)=q^{2}+1$ which appears in Example 5.3 i) maps the open ball $B=B(I, 1 / 2)$ centered at $I \in \mathbb{S}$ onto a non-open set: indeed $0 \in f(B)$ and $f(B) \cap L_{K} \subseteq \mathbb{R}$ when $K \in \mathbb{S}$ is orthogonal to $I$. Nevertheless we notice that $f(B(0, R))$ is open. In fact, if we choose an open set $U \subseteq B(0, R)$ which contains degenerate spheres for $f$, we can still prove that $f(U)$ is open provided $U$ has the following property.

Definition 6.4. Let $U \subseteq \mathbb{H}$. We say that $U$ is (axially) symmetric if, for all $x+y I \in$ $U$ with $y>0$, the whole 2-sphere $x+y \mathbb{S}$ is contained in $U$.

For all $x, y \in \mathbb{R}, r>0$ we may consider the neighborhood of $S=x+y \mathbb{S}$ defined by $C(S, r)=\{q \in \mathbb{H}: d(q, S)<r\}$ and notice that it is symmetric.

Theorem 6.5. Let $f: B(0, R) \rightarrow \mathbb{H}$ be a regular function. If $U$ is a symmetric open subset of $B(0, R)$, then $f(U)$ is open.

Proof. Let $p_{0} \in f(U)$. Choose $q_{0}=x_{0}+y_{0} I \in U$ such that $f\left(q_{0}\right)=p_{0}$. Since $f(q)-p_{0}$ has a zero on $S=x_{0}+y_{0} \mathbb{S} \subseteq U$, there exists $r>0$ such that $\overline{C(S, r)} \subseteq U$ and $f(q)-p_{0} \neq 0$ for all $q \in \overline{C(S, r)} \backslash S$. Let $\varepsilon>0$ be such that $\left|f(q)-p_{0}\right| \geq 3 \varepsilon$ for all $q$ such that $d(q, S)=r$. For all $p \in B\left(p_{0}, \varepsilon\right)$ we get

$|f(q)-p| \geq\left|f(q)-p_{0}\right|-\left|p-p_{0}\right| \geq 3 \varepsilon-\varepsilon=2 \varepsilon>\varepsilon \geq\left|p_{0}-p\right|=\left|f\left(q_{0}\right)-p\right|$

for $d(q, S)=r$. Thus $|f(q)-p|$ must have a local minimum point in $C(S, r)$. By the minimum modulus principle, there exists $q \in C(S, r)$ such that $f(q)-p=0$.

\section{References}

[1] F. Colombo, I. Sabadini, F. Sommen and D. C. Struppa, "Analysis of Dirac Systems and Computational Algebra", Vol. 39, Progress in Mathematical Physics, Birkhäuser Boston Inc., Boston, MA, 2004.

[2] C. G. CulLEN, An integral theorem for analytic intrinsic functions on quaternions, Duke Math. J. 32 (1965), 139-148.

[3] P. Dentoni and M. Sce, Funzioni regolari nell'algebra di Cayley, Rend. Sem. Mat. Univ. Padova 50 (1974), 251-267.

[4] R. Fueter, Die Funktionentheorie der differentialgleichungen $\Theta u=0$ und $\Theta \Theta u=0$ mit vier reellen variablen, Comment. Math. Helv. 7 (1934), 307-330. 
[5] R. FUETER, Über die analytische darstellung der regulären funktionen einer quaternionenvariablen, Comment. Math. Helv. 8 (1935), 371-378.

[6] G. GentiLi and C. Stoppato, Zeros of regular functions and polynomials of a quaternionic variable, Michigan Math. J. 56 (2008), 655-667.

[7] G. GentiLi and D. C. STRUPPA, A new approach to Cullen-regular functions of a quaternionic variable, C. R. Math. Acad. Sci. Paris 342 (2006), 741-744.

[8] G. Gentili and D. C. Struppa, A new theory of regular functions of a quaternionic variable, Adv. Math. 216 (2007), 279-301.

[9] K. I. KoU, T. QIAN and F. Sommen, Generalizations of Fueter's theorem, Methods Appl. Anal. 9 (2002), 273-289.

[10] V. V. Kravchenko and M. V. Shapiro, "Integral Representations for Spatial Models of Mathematical Physics", Vol. 351, Pitman Research Notes in Mathematics Series, Longman, Harlow, 1996.

[11] G. Laville and E. Lehman, Analytic Cliffordian functions, Ann. Acad. Sci. Fenn. Math. 29 (2004), 251-268.

[12] G. LAVILlE and I. RAmadanofF, Holomorphic Cliffordian functions, Adv. Appl. Clifford Algebr. 8 (1998), 323-340.

[13] A. Pogorui and M. Shapiro, On the structure of the set of zeros of quaternionic polynomials, Complex Var. Theory Appl. 49 (2004), 379-389.

[14] T. QIAN, Generalization of Fueter's result to $\mathbf{R}^{n+1}$, Atti Accad. Naz. Lincei Cl. Sci. Fis. Mat. Natur. Rend. Lincei (9) Mat. Appl. 8 (1997), 111-117.

[15] M. SCE, Osservazioni sulle serie di potenze nei moduli quadratici, Atti Accad. Naz. Lincei. Rend. Cl. Sci. Fis. Mat. Nat. (8) 23 (1957), 220-225.

[16] A. Sudbery, Quaternionic analysis, Math. Proc. Cambridge Philos. Soc. 85 (1979), 199-224.

Dipartimento di Matematica "U. Dini" Università di Firenze

Viale Morgagni 67/A

50134 Firenze, Italia gentili@math.unifi.it stoppato@math.unifi.it 